\title{
用酵母双杂交系统分离一个新的 人类细胞凋亡诱发基因*
}

\section{齐 兵 ${ }^{(1)}$ 齐义鹏 ${ }^{(1 * *}$ M. Yutsudo ${ }^{(2)}$ 刘青珍 ${ }^{(1)}$}

(1)武汉大学病毒学研究所, 武汉 430072; (2)Research Institute for Microbial Diseases, Osaka University, Osaka 565, Japan)

\begin{abstract}
摘要 asy 基因是最近发现的一个新的调亡诱发基因, 但其诱发调亡的机制仍不清楚. 采用酵母双杂交系统，从人肺细胞系(WI-38)cDNA 文库中克隆了一个 asy 相互作用蛋 白基因 asyip (asy interacting protein). asyip 在人的各种组织内均能组成型转录合成 1.8 和 $2.7 \mathrm{~kb}$ 两种 $\mathrm{mRNA}$ 转录本, 但转录水平有明显差异, 在脑组织中转录水平最高. 而 且, 脑组织中 $2.7 \mathrm{~kb} \mathrm{mRNA}$ 转录水平大大高于 $1.8 \mathrm{~kb}$ 的转录本. 序列分析表明两种转 录本起始于一个共同的 $5^{\prime}$ 端, 但 $3^{\prime}$ 端加 poly(A)位点不同. 两者共享一个大的可读框, 编码 $26 \mathrm{ku}$ 的蛋白 ASYIP. 推论的氨基酸一级结构表明, ASYIP 含有两个大的强疏水 区、两个蛋白激酶 C 磷酸化位点和两个酪蛋白激酶磷酸化位点. C 端具有一个内质网 捕获信号(Lys-Lys-Lys-Ala-Glu). 免疫沉淀试验进一步验证 ASYIP 和 ASY 在哺乳动物 细胞中可以相互结合. 与 asy 基因一样, asyip 基因的大量表达能抑制肿瘤细胞 Saos-2 的生长, 诱发细胞凋亡, 但效率比 asy 低.
\end{abstract}

\section{关键词 asy 基因 asyip 基因 细胞调亡 酵母双杂交系统 人肺细胞 cDNA 文库}

细胞程序性死亡或细胞调亡是细胞的基本生命特征, 它在胚胎发育、细胞衰老死亡、机 体内环境稳定、细菌和病毒感染细胞的清除过程中起着重要作用. 许多疾病如肿瘤、Alzheimer 病(AD)的发生与细胞调亡失控有关. 细胞凋亡可被多种因素诱发, 凋亡细胞均表现共同的形 态学、生物化学特征, 包括胞体坡缩、体积缩小、细胞核浓缩、染色体 DNA 断裂等等 ${ }^{[1]}$. 到 目前为止, 与细胞调亡诱发、调控过程相关的许多蛋白因子已被鉴定 ${ }^{[1]}$, 两条经典的细胞调亡 途径也已被阐明. 一条途径由发育信号或生长因子撤除所诱发, 引发细胞色素 C 从线粒体中 释放, 使 Apaf-1(apoptotic protease activating factor-1)多聚化和 Caspase-9 被激活, 此途径可被 Bcl-2 家族蛋白所调控 ${ }^{[1,2]}$. 另一条途径由细胞死亡受体(TNFR 或 Fas)介导, 通过 FADD (Fasassociating death domain protein)和 FLASH(FLICE-associated huge)招募和激活 Caspase- $8^{[1,3]}$. 两 条途径最终均激活 Caspase-3, Caspase-3 再活化 Caspase-激活的脱氧核糖核酸酶 (caspaseactivated deoxyribonuclease, CAD), 引起染色体 DNA 断裂 $[4,5]$.

asy (apoptosis/saibousi yuudou)基因是最近发现的一个调亡诱发基因 ${ }^{[6]}$. 研究表明, 它在多 种肿瘤细胞中并没有发生突变, 其 mRNA 转录水平和蛋白质表达水平与正常细胞也没有显著 差异. 采用人巨细胞病毒 HCMV 强启动子在细胞内瞬时高效表达 asy 基因能迅速激活 Caspase-3,

** 联系人(E-mail: mvlwh@whu.edu.cn) 
诱发细胞凋亡，但具体机制仍不明了.

酵母双杂交系统是目前研究蛋白质相互作用的一种新型系统 ${ }^{[7]}$. 它利用酵母转录激活蛋 白 Gal4 的两个相互独立功能区: DNA 结合功能区(DNA binding domain, DBD)和转录激活功 能区 (activation domain, AD), 将已知的 X 蛋白和另一种未知的(或已知的) Y 蛋白分别与 DBD 和 $\mathrm{AD}$ 融合. 如果 $\mathrm{X}$ 蛋白和 $\mathrm{Y}$ 蛋白能相互结合, 则 $\mathrm{DBD}$ 和 $\mathrm{AD}$ 在空间上将足够靠近并产生 具有功能的转录激活蛋白, 从而激活 gal4 基因启动子, 驱动报告基因的表达. 如果 X 蛋白和 $\mathrm{Y}$ 蛋白不能结合，报告基因的转录则不能被激活. 因此，该系统通过检测报告基因是否被转录 来证明蛋白质之间的相互作用或分离能与已知基因相互作用的新基因.

为研究 asy 基因的调亡诱发机制，阐明 asy 诱发细胞调亡的途径，我们采用酵母双杂交系 统, 以 ASY 为诱饵蛋白(bait), 从人肺细胞系(WI-38)cDNA 文库中克隆了一个新的基因 asyip (asy interacting protein), 其编码的蛋白与 ASY 在酵母和哺乳动物细胞内均能相互结合. 与 asy 基因一样, asyip 在细胞内的高效表达也能抑制人骨肉瘤细胞系 Saos-2 生长、诱发细胞调亡.

\section{1 材料和方法}

\section{1 细胞、基因、cDNA 文库和载体}

$\mathrm{HeLa}$ 细胞和人正常纤维细胞的杂交细胞系 CGL1 在 DMEM 培养液(加 $10 \%$ 胎牛血清)中 培养, 人骨肉瘤细胞系 Saos-2 在 1640 培养液(加 10\%胎牛血清)中培养. asy 基因与以上两种 细胞均由日本 Osaka 大学 M. Yutsudo 博士提供. 酵母双杂交系统试剂盒(质粒 pGBT9, pGAD424, pLAM5' 和酵母菌株 HF7C)购于 Clontech 公司. pGBT9 质粒含 trpl 基因和 gal4 DNA 结合功能区 (gal4-DBD), 含此质粒的转化子能在 $\mathrm{Trp}^{-}$培养基中生长; pGAD424 质粒含 leu2 基因和 gal4 激活功能区 (gal4-AD), 含此质粒的转化子能在 $\mathrm{Leu}^{-}$培养基中生长; pLAM5' 质 粒含 $\operatorname{trpl}$ 基因，能表达 gal4-DBD 与核纤层蛋白 C(lamin C)的融合蛋白. 以 $\mathrm{pACT} 2$ 载体(含 leu2 基因和 gal4-AD)构建的人肺细胞系(WI-38)MATCHMAKER cDNA 文库购于 Clontech 公司. 本 文使用和构建的各种载体的结构见图 1.

pGBT9
pGBTasy
pGAD424
pGADasyip
pHM6
pHMasyip
pCFasy
pCMV-NEO-BAM
pCMVasy
pCMVasyip

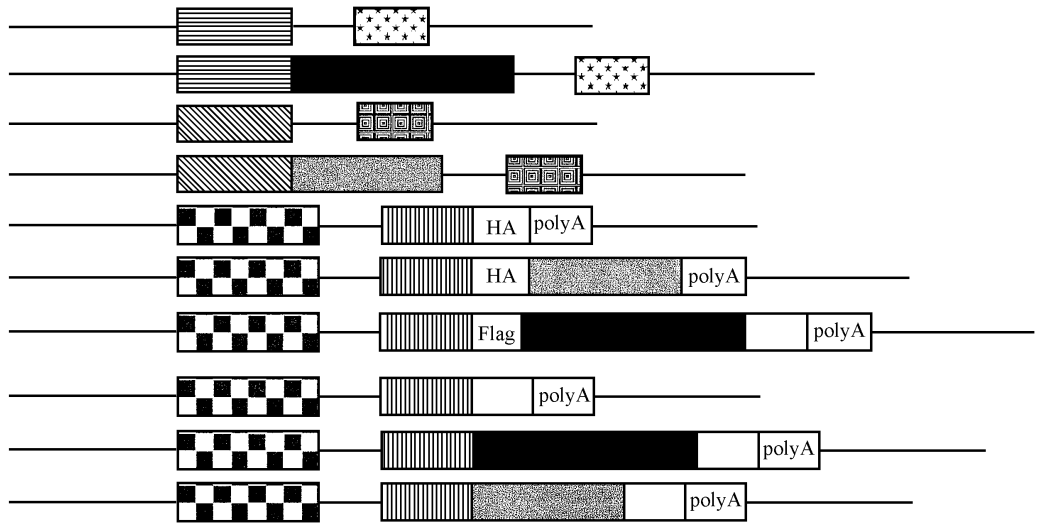

asy 基因

asyip 基因

产采 gal4-DBD

*** $\operatorname{trp} 1$ 基因

MIIU gal4-AD

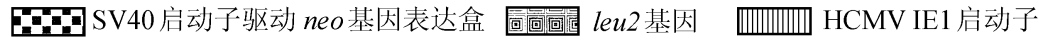
内含子

图 1 文中使用的各种载体的结构示意图 


\section{2 酵母双杂交系统}

将 asy 基因的可读框插入 pGBT9 载体，与 gal4 DNA 结合功能区融合并保持顺框，构建 成表达载体 pGBTasy(图 1). 采用标准 LiAC 方法将 pGBTasy 质粒与人肺细胞系(WI-38)cDNA 文库共转化酵母菌株 $\mathrm{HF} 7 \mathrm{C}$, 铺于 $\mathrm{SD} / \mathrm{Trp}^{-} \mathrm{Leu}^{-} \mathrm{His}^{-}$营养缺陷型培养基上培养, 7 10 d 后挑取 可能的阳性转化子进行半乳糖苷酶活力滤膜试验. 酵母双杂交系统实验操作根据 Clontech 公 司 MATCHMAKER GAL4 双杂交用户操作手册进行.

\subsection{Northern 分析}

$2 \mu \mathrm{g}$ 来源于人各种组织的 poly(A) RNA 被固定在 MTN(Multiple Tissue Northern)膜 (Clontech 公司产品)上, 以 $\alpha-{ }^{32} \mathrm{P}$ 标记酵母双杂交系统得到的阳性克隆 cDNA 片段作分子杂交 探针, 与 poly(A) RNA 杂交, Northern 分析采用标准分子克隆操作 ${ }^{[8]}$ 进行.

\section{4 菌落原位杂交}

以人正常纤维细胞系 TIG3 cDNA 文库(日本 Osaka 大学 NorJima 博士赠送)用于全长 cDNA 的䇻选和克隆，以 $\alpha{ }^{32} \mathrm{P}$ 标记阳性克隆中 cDNA 的 Sal I /Pst I 片段作分子杂交探针，采用标 准分子克隆操作进行菌落原位杂交 ${ }^{[8]}$.

\section{5 序列测定及计算机分析}

采用双脱氧链终止法和 SequiTherm EXCETM Long-ReadTM Cycle 苂光测序试剂盒 (Epicentre Technologies 公司产品)测定 DNA 序列. DNA 和氨基酸序列的计算机分析采用 GENETYX9.0 软件.

\section{6 免疫沉淀试验}

将 asy 基因可读框与十二肽 Flag(Sigma 公司产品)编码框融合，插入 pCMV-NEO- BAM 载体(美国 John Hopkinse 大学 Bent Vogelstein 博士赠送), 构建成 Flag-ASY 融合蛋白表达载 体 pCFasy; 将 asyip 基因可读框插入 pHM6(Boehringer Mannheim 公司产品)表达载体, 和血 细胞凝集素 HA 短肽融合并保持顺框，构建成 HA-ASYIP 融合蛋白表达载体 pHMasyip(图 1). 每种质粒 DNA $1 \mu \mathrm{g}$ 以 Lipofect AMINE PLUS 试剂(Life Technologies 公司产品)共转染 CGL1 细胞, $19 \mathrm{~h}$ 后, 收集细胞并在 NP40 缓冲液 $\left(142.5 \mathrm{mmol} / \mathrm{L} \mathrm{KCl}, 5 \mathrm{mmol} / \mathrm{L} \mathrm{MgCl}_{2}, 10 \mathrm{mmol} / \mathrm{L}\right.$ HEPES(pH 7.2), $1 \mathrm{mmol} / \mathrm{L}$ EDTA, 0.25\% NP-40, $1 \mathrm{mmol} / \mathrm{L}$ PMSF, $10 \mathrm{mg} / \mathrm{mL}$ aprotinin)中温和裂 解. Flag-ASY 免疫沉淀物和 HA-ASYIP 免疫沉淀物分别用单克隆抗体 anti-Flag(Sigma 公司产 品) 或 anti-HA(Boehringer Mannheim 公司产品)和偶联有蛋白 A/G PLUS 的琼脂糖(Santa Cruz 公司产品)从裂解细胞中沉淀出来. 采用单克隆抗体 anti-Flag 或 anti-HA 和 ECL Western 显色 试剂(Amersham 公司产品)进行免疫沉淀物的 Western blot 分析.

\subsection{G418 抗性细胞集落形成试验}

asy 基因全长 cDNA, asyip 基因 $1.8 \mathrm{~kb}$ cDNA 片段分别插入 pCMV-NEO-BAM 载体，构建 成 pCMVasy 和 pCMVasyip 两个表达载体(图 1). 以 Xba I 分别消化表达载体 pCMV-NEO- BAM, pCMVasy 和 pCMVasyip, 然后用 Lipofect AMINE PLUS 试剂(Life Technologies 公司产品)转 染生长在 $60 \mathrm{~mm}$ 细胞培养板上 $50 \%$ \% 0 \%丰度的 Saos-2 细胞, $24 \mathrm{~h}$ 后, 转染细胞被转至 $100 \mathrm{~mm}$ 细胞培养板上, 在含 $300 \mu \mathrm{g} / \mathrm{mL} \mathrm{G} 418$ 的 1640 细胞培养液中培养. G418 篮选 $14 \mathrm{~d}$ 后, 以 $50 \%$ 甲醇固定和晶体蓝染色并计数 G418 抗性的细胞集落. 


\subsection{TdT 酶介导 dUTP-X 缺口末端标记(TUNEL)试验}

将 50\%丰度的 Saos-2 细胞培养在无菌的玻璃载玻片上, 分别取 $0.15 \mu \mathrm{g}$ 的 pCMV-NEOBAM, pCMVasy 和 pCMVasyip 质粒 DNA 以 Lipofect AMINE PLUS 试剂(Life Technologies 公 司产品)转染细胞. $48 \mathrm{~h}$ 后, 用 $4 \%$ 低聚甲醛于 $4{ }^{\circ} \mathrm{C}$ 固定 $20 \mathrm{~min}$, PBS 洗涤 3 遍, 然后用 $0.1 \%$ Triton X-100 室温处理 5 min, 再用 PBS 洗涤 3 遍. TUNEL 试验采用 Apoptag Direct 原 位调亡检测试剂盒(Oncor, Gaihersburg, MD 公司产品), 按照试剂盒说明书标准步骤进行操作.

\section{2 结果}

\section{1 asyip 基因全长 cDNA 的克隆和鉴定}

将 asy 基因的可读框插入 pGBT9 质粒，构建成表达质粒 pGBTasy(图 1)，asy 与 gal4 DNA 结合功能区融合并在酵母细胞中表达，作为诱饵蛋白(bait).pGBTasy 和人肺细胞系(WI-38) cDNA 文库共转化酵母菌 HF7C 后, 从 $1 \times 10^{6}$ 个共转化子中分离鉴定了 3 个 $\mathrm{His}^{+} \mathrm{LacZ}^{+}$克隆. 序 列分析表明其中两个克隆的 cDNA 片段编码 ASY C 端多肽, 而另一个克隆的 cDNA 片段与 GenBank 全序列比较没有发现同源基因，推测是一个新基因，命名为 asy 相互作用蛋白基因 asyip (asy interacting protein).

采用菌落原位杂交方法，从人正常纤维细胞 TIG3 cDNA 文库中篮选 asyip 基因的全长 cDNA，分离了与 asyip 探针有杂交信号的 9 个克隆，其中 4 个克隆插入片段大约为 $2.5 \mathrm{~kb}$ ，对 应于 $2.7 \mathrm{~kb}$ 的转录本; 5 个克隆插入片段大约为 $1.6 \mathrm{~kb}$, 对应于 $1.8 \mathrm{~kb}$ 的转录本. 对 9 个克隆 中的插入片段进行序列分析, 结果表明, 1.8 和 $2.7 \mathrm{~kb}$ 两种转录本起始于共同的 $5^{\prime}$ 端, 终止在 不同的 $3^{\prime}$ 端，暗示两种转录本均由同一个启动子驱动转录，但加 poly(A)的位点不同. asyip 基 因全长 cDNA 序列见图 2, 从图 2 看出，在 1663,1694 和 $2510 \mathrm{nt}$ 处存在 3 个加 poly(A)信号 (AATAAA).

asyip 基因 cDNA 全长 $2537 \mathrm{bp}, 2.7$ 和 $1.8 \mathrm{~kb}$ 两种转录本具有一个共同的可读框，基因的 起始密码子侧翼序列(GCCATGG)符合 Kozak 规则 ${ }^{[9]}$, 能编码一个 236 个氨基酸的多肽, 分子 量约为 $26 \mathrm{ku}$. 推测的 ASYIP 氨基酸序列含有两个蛋白激酶 C 磷酸化位点 (SFR 在 aa96 98, SLK 在 aa161 163), 两个酪蛋白激酶磷酸化位点(TQID 在 aa203 206, SIVE 在 aa218 221)(图 2). ASYIP 蛋白的亲疏水性分析表明该蛋白含有两个大的强疏水区 (aa60 99 和 aa148 196), 可 能为潜在的跨膜区, $C$ 端含一个内质网捕获信号 (Lys-Lys-Lys-Ala-Glu), 其 C 端 -3 和- 4 或 -5 位为赖氨酸 ${ }^{10]}$. ASYIP 和 ASY 的 C 端氨基酸序列有高度同源性(56.60\%)，在 ASYIP 氨基酸 88 120 区和 151 187 区同源性更高，达 88\%和 81\%。

\section{2 asyip 基因在人的各种组织中的表达}

Northern 杂交结果(图 3)显示, asyip 基因在人各种组织中均组成性转录产生 1.8 和 $2.7 \mathrm{~kb}$ 两种 mRNA 转录本, 但转录水平在不同组织中有一定差异, 在胰、肌肉、白细胞、结肠、小 肠和睪丸中转录水平较高, 在脑组织中转录水平最高. 特别是胰和睪丸的 $1.8 \mathrm{~kb} \mathrm{mRNA}$ 转录 水平高于其他组织, 而脑组织中 $2.7 \mathrm{~kb} \mathrm{mRNA}$ 的转录水平不仅大大高于 $1.8 \mathrm{~kb}$ 的转录本, 也 高于其他组织.

\section{3 两种蛋白 ASYIP 与 ASY 在酵母中相互作用的验证}

为验证 ASYIP 与 ASY 在酵母中的相互作用，将 asyip 基因的可读框插入 pGAD424 表达 
TCTGTCGGAGTCTGTCCTCGGAGCAGGCGGAGTAAAGGGACTTGAGCGAGCCAGTTGCCGGATTATTCTATTTCCOCTCCCTCTCTCCCG

90

ССССGTATCTCTTTTCACСCTTCTCCСACСCTCGCTCGCGTA GCCATGGCGGAGCCGTCGGCGGCCACTCAGTCCCATTCCATCTCCTCG 180 $\begin{array}{lllllllllllllllll}\text { M } & A & E & P & S & A & A & T & Q & S & H & S & \text { I } & S & S & & 15\end{array}$

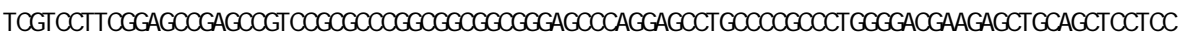

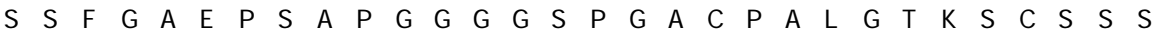
TGTGCGGTGCACGATCTGATTTTCTGGAGAGATGTGAAGAAGACTGGGTTTGTCTTTGGCACCACGCTGATCATGCTGCTTTCCCTGGCA

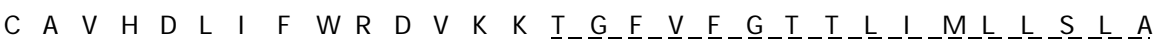
GCTTTCAGTGTCATCAGTGTGGTTTCTTACCTCATCCTGGCTCTTCTCTCTGTCACCATCAGCTTCAGGATCTACAAGTCCGTCATCCAA

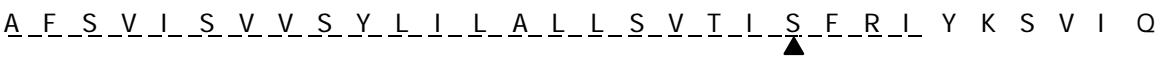
GCTGTACAGAAGTCAGAAGAAGGCCATCCATTCAAAGCCTACCTGGACGTAGACATTACTCTGTCCTCAGAAGCTTTCCATAATTACATG

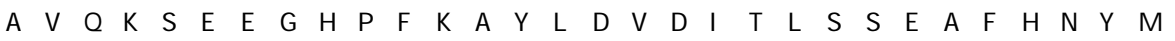
AATGCTGCCATGGT GCACATCAACAGGGCCCTGAAACTCATTATTCGTCTCTTTCTGGTAGAAGATCT GGTTGACTCCTTGAAGCTGGCT

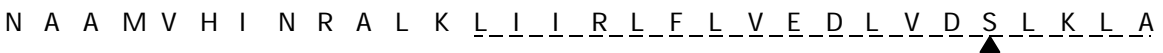
GTCTTCATGTGGCTGATGACCTATGTTGGTGCTGTTTTTAACGGAATCACCCTTCTAATTCTTGCTGAACTGCTCATTTTCAGTGTCCCG

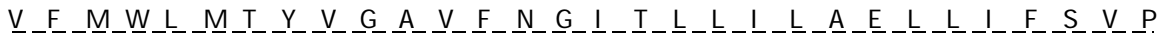
ATTGTCTATGAGAAGTACAAGACCCAGATTGATCACTATGTTGGCATCGCCCGAGATCAGACCAAGTCAATTGTTGAAAAGATCCAAGCA I V V AAACTCCCTGGAATCGCCAAAAAAAAGGCAGAATAAGTACATGGAAACCAGAAATGCAACAGTTACTAAAACACCATTTTAATAGTTATAA

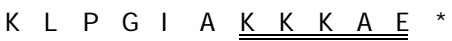
CGTCGTTACTTGTACTATGAAGGAAAATACTCAGTGTCAGCTTGAGCCTGCATTCCAAGCTTTTTTTTTTAATTTGGTGTTTTCTCCCATC CTTTССCTTTAAССCTCAGTATCAAGCACAAAAATTGATGGACTGATAAAAGAACTATCTTAGAACTCAGAAGAAGAAAGAATCAAATTC ATAGGATAAGTCAATACCTTAATGGTGGTAGAGCCTTTACCTGTAGCTTGAAAGGGGAAAGATTGGAGGTAAGAGAGAAAATGAAAGAAC ACCTCTGGGTCCTTCTGTCCAGTTTTCAGCACTAGTCTTACTCAGCTATCCATTATAGTTTTGCCCTTAAGAAGTCATGATTAACTTATG AAAAAATTATTTGGGGACAGGAGTGTGATACCTTCCTTGGTTTTTTTTTTGCAGCCCTCAAATCCTATCTTCCTGCCCCACAATGTGAGCA GCTACCCCTGATACTCCTTTTCTTTAATGATTTAACTATCAACTTGATAAATAACTTATAGGTGATAGTGATAATTCCTGATTCCAAGAA TGCCATCTGATAAAAAAGAATAGAAATGGAAAGTGGGACTGAGAGGGAGTCAGCAGGCATGCTGCGGTGGCGGTCACTCCCTCTGCCACT ATCCCCAGGGAAGGAAAGGCTCCGCCATTTTGGGAAAGTGGTTTCTACGTCACTGGACACCGGTTCTGAGCATTAGTTTGAGAACTCGTTC ССGAATGTGCTTTССTСССTCTССССTGCССАССTCAAGTTTAATAAATAAGGTTGTACTTTTCTTACTATAAAATAAATGTCTGTAACT GCTGTGCACTGCTGTAAACTTGTTAGAGAAAAAAATAACCTGCATGTGGGCTCCTCAGTTATTGAGTTTTTGTGATCCTATCTCAGTCTG GGGGGGAACATTCTCAAGAGGTGAAATACAGAAAGCCTTTTTTTTCTTGATCTTTTCCCGAGATTCAAATCTCCGATTCCCATTTGGGGGC AAGTTTTTTTCTTCACCTTCAATATGAGAATTCAGCGAACTTGAAAGAAAAATCATCTGTGAGTTCCTTCAGGTTCTCACTCATAGTCAT GATCCTTCAGAGGGAATATGCACTGGCGAGTTTAAAGTAAGGGCTATGATATTTGATGGTCCCAAAGTACGGCAGCTGCAAAAAGTAGTG GAAGGAAATTGTCTACGTGTCTTGGAAAATTAGTTAGGAATTTGGATGGGTAAAAGGTACCCTTGCCTTACTCCATCTTATTTTCTTAG CCCCCTTTTGAGTGTTTTAACTGGTTTCATGTCCTAGTAGGAAGTGCATTCTCCATCCTCATCCTCTGCCCTCCCAGGAAGTCAGTGATTG TCTTTTTGGGCTTCCCCTCCAAAGGACCTTCTGCAGTGGAAGTGCCACATCCAGTTCTTTTCTTTTGTTGCTGCTGTGTTTAGATAATTG AAGAGATCTTTGTGCCACACAGGATTTTTTTTTTTTTTTAAGAAAAACCTATAGATGAAAAATTACTAATGAAACTGTGTGTACGTGTCTG TGCGTGCAACATAAAAATACAGTAGCACCTAAGGAGCTTGAATCTTGGTTCCTGTAAAATTTCAAATTGATGTGGTATTAATAAAAAA( A) n 2537

图 2 asyip 基因 $2.5 \mathrm{~kb}$ 全长 cDNA 核苷酸序列和推导的氨基酸序列

起始密码子及 Kozak 序列用方框标示, 3 个 poly(A)加尾信号用下划单线标明, 两个大的疏水区用下划虚线标明, 内质网 捕获信号(KKKAE)用下划双线标明. 蛋白激酶 C 磷酸化位点和酪蛋白激酶 II磷酸化位点分别用(
270

45 360 75 450 105 540 135 630 165 720 195 810 225 900 236 990 1080 1170 1260 1350 1440 1530 1620 1710 1800 1890 1980 2070 2160 2250 2340 2430 


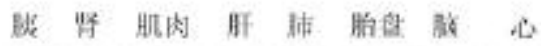
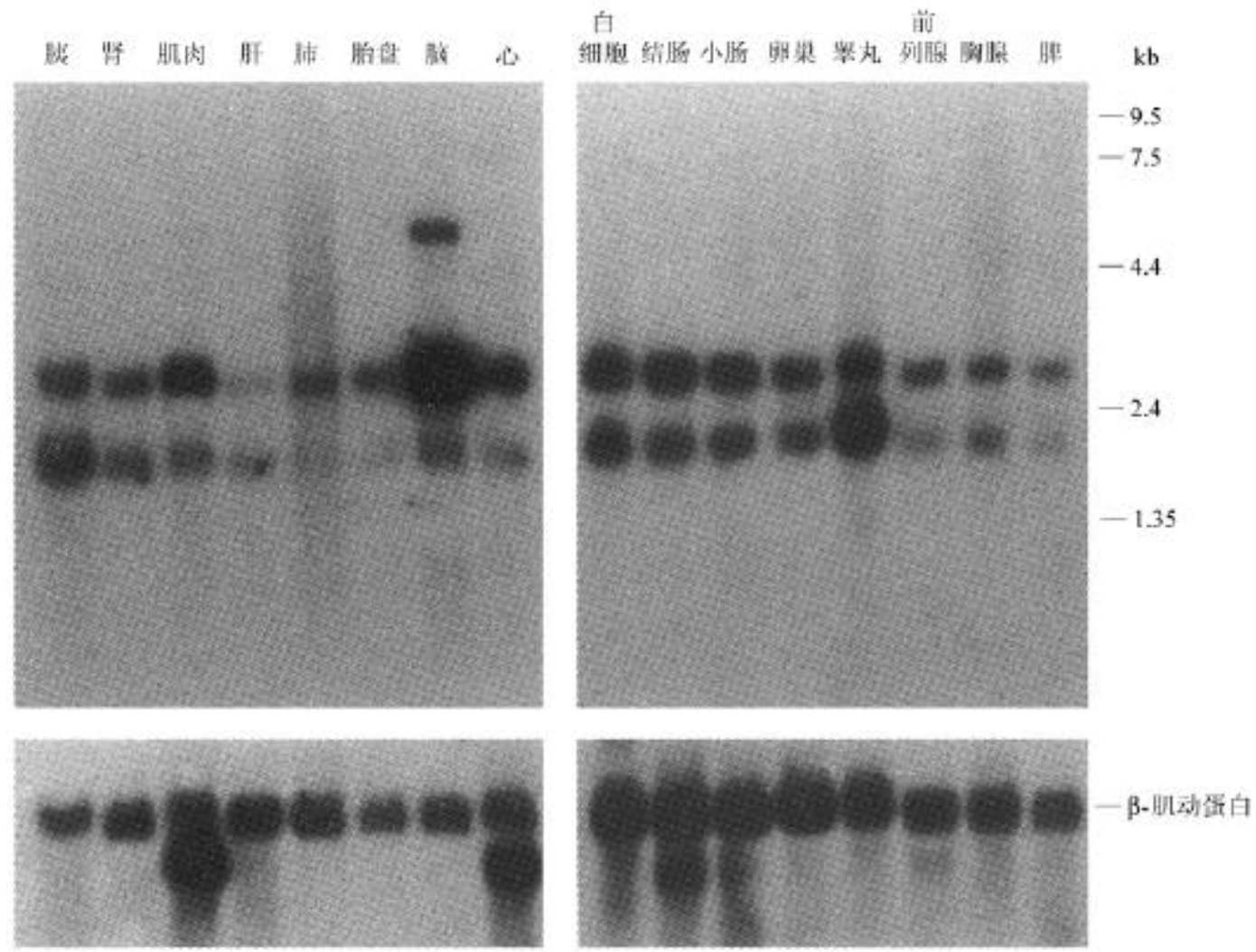

图 3 Northern 杂交显示 asyip 基因在人各种组织内的表达

显示 2.7 和 $1.8 \mathrm{~kb}$ 两种转录本, 其中脑组织中转录水平最高(特别是 $2.7 \mathrm{~kb}$ 的 mRNA). $\beta$-肌动蛋白 mRNA 转录水平作为对照

载体, 构建成 Gal4-AD/ASYIP 融合蛋白表达载体 pGADasyip(图 4(a)), 将它与 Gal4-DBD/ASY 融合蛋白表达载体 pGBTasy 共转化酵母菌 HF7C. 如图 4(b)所示, 共表达两种融合蛋白 Gal4AD/ASYIP 和 Gal4-DBD/ASY 的酵母 HF7C 能很好地生长在含 $3 \mathrm{mmol} / \mathrm{L} \mathrm{3-AT}$ 的 SD/Leu ${ }^{-} \mathrm{Trp}$ ${ }^{-}$His ${ }^{-}$营养缺陷型培养基上, 但共表达融合蛋白 Gal4-AD/ASYIP 和 Gal4-DBD 或 Gal4$\mathrm{DBD} / \mathrm{LAM} 5^{\prime}$ 的酵母仅能在 SD/Leu ${ }^{-} \mathrm{Trp}^{-}$培养基上生长, 不能在含 $3 \mathrm{mmol} / \mathrm{L} 3-\mathrm{AT}$ 的 SD/Leu ${ }^{-} \mathrm{Trp}^{-} \mathrm{His}^{-}$培养基上生长. $\beta$-半乳糖苷酶活力滤膜试验也证明了 pGADasyip 和 pGBTasy 共转 化的 HF7C 能激活 $l a c Z$ 基因的表达(图 4(c)). 由图 4 可以得出以下结论: 含有质粒 pGADasyip 和 pGBTasy 的酵母菌 HF7C 能表达两种融合蛋白 Gal4-AD/ASYIP 和 Gal4-DBD/ASY, 由于 ASYIP 和 ASY 在酵母中的相互作用, 两个融合蛋白形成了包含 Ga14-AD 和 Gal4-DBD 的转 录激活复合物, 从而激活了 gal4 启动子驱动的 his 基因和 lacZ 基因的表达, 使酵母 HF7C 转 化子具有 $\mathrm{His}^{+}$和 $\mathrm{LacZ}^{+}$的表型.

\section{4 两种蛋白 ASYIP 与 ASY 在哺乳动物细胞中的相互作用}

为进一步研究 ASYIP 与 ASY 在哺乳动物细胞中的相互作用, 以表达融合蛋白 Flag-ASY 的表达载体 pCFasy 和表达融合蛋白 HA-ASYIP 的表达载体 pHMasyip 共转染 CGL1 细胞. 细 胞抽提物用单克隆抗体 anti-Flag 或 anti-HA 免疫沉淀, 然后再用 anti-Flag 或 anti-HA 为探针 进行 Western blot 杂交. 如图 5 所示, HA-ASYIP 能在单克隆抗体 anti-Flag 的免疫沉淀物中检 


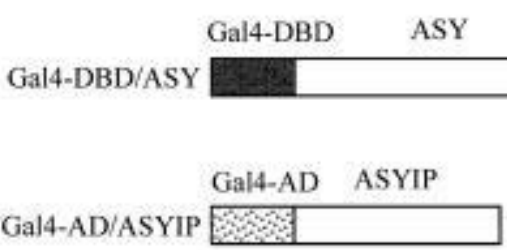

(a)

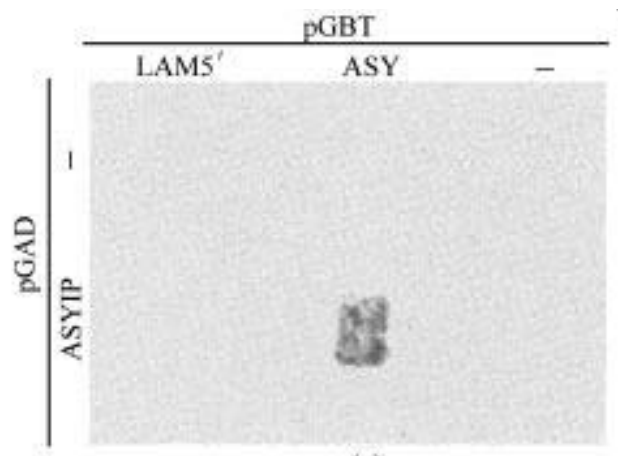

(c)
SD/Trp Leu'His

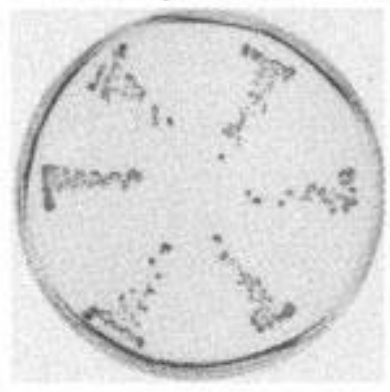

SD/Trp Leu His' (3 mmol/L 3-AT)

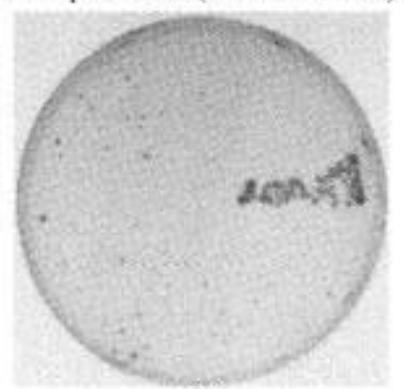

(b)

图 4 ASYIP 和 ASY 在酵母中的相互作用

(a) 融合蛋白 Gal4-DBD/ASY 和 Ga14-AD/ASYIP 的结构. (b) 酵母 HF7C 共转化子在选择性培养基上的生长. 3-AT 为

3-氨基 1,2,4-三唑; LAM5' 为核纤层蛋白 C; GAl4-AD 和 GAl4-DBD 为阴性对照. (c) $\beta$-半乳糖苷酶活力滤膜试验

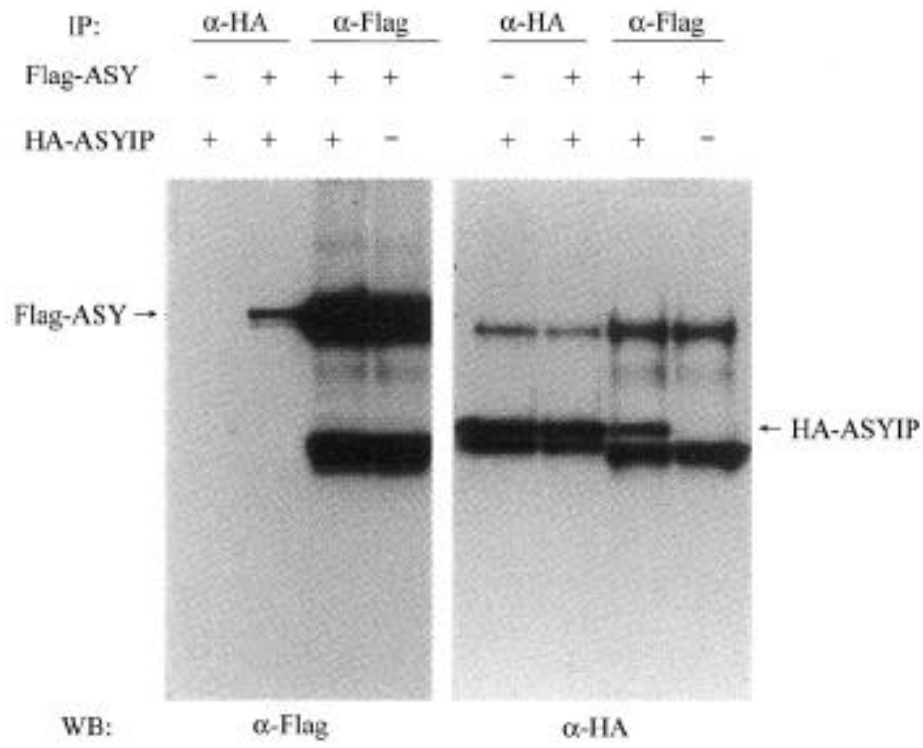

图 5 ASYIP 和 ASY 在哺乳动物细胞内的相互作用

Flag-ASY 融合蛋白能在单克隆抗体 $\alpha$-HA 免疫沉淀物中检测出来, HA-ASYIP 融合蛋白能在单克隆抗

体 $\alpha$-Flag 免疫沉淀物中检测出来. IP 为免疫沉淀; WB 为 Western blotting 
测出来, 而 Flag-ASY 也能在单克隆抗体 anti-HA 的免疫沉淀物中检测出来, 证明两种表达蛋 白 ASYIP 和 ASY 在哺乳动物细胞中也能发生相互作用.

\subsection{ASYIP 对肿瘤细胞生长的抑制}

为检测 ASYIP 是否具有与 ASY 同样的细胞生长抑制功能, 以 HCMV 启动子驱动 asy 基 因或 asyip 基因表达, 构建了表达载体 pCMVasy 和 pCMVasyip (图 1). 为避免 $2.7 \mathrm{~kb}$ 的 asyip cDNA $3^{\prime}$ 端非翻译区对蛋白表达的影响, 选用 $1.8 \mathrm{~kb}$ 的 asyip cDNA 作为插入片段. pCMVasy, pCMVasyip 和对照载体 pCMV-NEO-BAM 分别转染 Saos-2 细胞, G418 篮选 $14 \mathrm{~d}$ 后, 细胞集 落被固定、染色. 细胞集落计数发现，对照载体转染得到的细胞集落约为 asy 表达质粒转染 的 42 倍, 而 asyip 表达质粒转染产生的细胞集落仅为对照载体转染的 32\%(图 6(a)). 结果证明, asy 和 asyip 的大量表达能抑制肿瘤细胞 Saos-2 的生长, 但 ASYIP 的抑制效率明显低于 ASY.

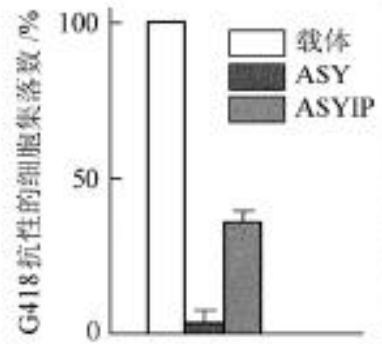

(a)

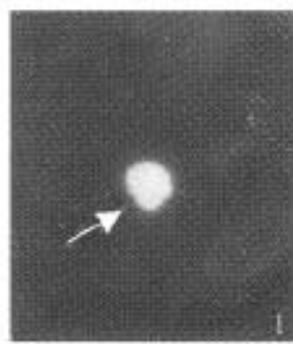

图 6 ASY 和 ASYIP 对肿瘤细胞 Saos-2 的生长抑制及调亡诱发

(a) G418 抗性细胞集落形成试验. (b) TUNEL 试验检测细胞凋亡. 1 为以表达载体 pCMVasy 转染的 Saos-2 细胞, 箭 头所指为调亡细胞核的苂光信号; 2 为以表达载体 pCMVasyip 转染的 Saos-2 细胞, 箭头所指为凋亡细胞核的苂光 信号; 3 为以对照载体 pCMV-NEO-BAM 转染的 Saos-2 细胞

\subsection{ASYIP 诱发细胞凋亡}

为了阐明 ASYIP 对细胞生长的抑制是否由于在细胞内诱发了细胞凋亡，以 pCMV- NEOBAM, pCMVasy, pCMVasyip 分别转染 Saos-2 细胞, $48 \mathrm{~h}$ 后, 用末端脱氧核苷酸转移酶(TdT)和 苂光标记的核苷酸检测细胞的 DNA $3^{\prime}$ 末端. 图 6(b)显示在表达质粒 pCMVasy 和 pCMVasyip 转染的 Saos-2 细胞内检测到调亡细胞中浓缩细胞核的苂光信号, 而对照载体转染的 Saos-2 细 胞内没有检测到苂光信号, 证明 asy 和 asyip 在细胞内高效表达能引起染色体 DNA 断裂, 诱 发细胞调亡.

\section{3 讨论}

酵母双杂交系统作为研究蛋白质相互作用的一种新的强有力技术，目前已被广泛应用于 生物学研究各个领域 ${ }^{[7]}$. 在细胞信号传导、细胞周期调控、基因转录和翻译调控等各个方面, 利 用酵母双杂交系统已经克隆了许多重要的新基因(如 FLASH ${ }^{[11]}$ ，TRADD(TNFR1-associated death domain protein $)^{[12]}$ 等). 我们采用酵母双杂交系统, 以新的人细胞凋亡诱发基因 ASY ${ }^{[6]}$ 为 诱饵蛋白, 从人肺细胞系(WI-38) cDNA 文库内分离克隆 ASY 相互作用蛋白基因. 以 pGBTasy 表达质粒和 cDNA 文库共转化酵母菌 HF7C 后, 鉴定了 3 个 $\mathrm{His}^{+} \mathrm{LacZ}^{+}$克隆, 序列分析结果显 示, 其中两个克隆的 cDNA 片段编码 ASY C 端多肽, 表明 ASY 在酵母内具有形成同源二聚 体或多聚体的能力. 另外一个克隆的 cDNA 片段在 GenBank 内未发现同源基因, 命名为 asy 
相互作用蛋白基因(asy interacting protein, asyip).

asyip 基因 cDNA 全长 $2.5 \mathrm{~kb}$, 在人的各种组织内都能转录 2.7 和 $1.8 \mathrm{~kb}$ 两种转录本, 其 转录水平在不同组织内也有一定差异. 有趣的是, 在高度分化的脑组织中, asyip 转录水平最 高, $2.7 \mathrm{~kb}$ 转录本的转录水平也大大高于 $1.8 \mathrm{~kb}$ 的转录水平. 由于两种转录本使用的是同一 个启动子, 两种 mRNA 的合成是由于使用了不同的 poly(A)加尾信号所致, 暗示在脑组织中存 在某种特别的机制, 使 asyip 基因的转录能越过第 1 和第 2 个 poly(A)信号, 优先使用了第 3 个 poly(A)信号. 目前, $3^{\prime}$ 非翻译区(UTR)对基因转录、翻译的调控屡见报道 ${ }^{[13]}$. Sauvage 等人 ${ }^{[14]}$ 指出, 淀粉状蛋白前体 mRNA 也存在两个 poly(A)信号, 而两个 poly(A)信号之间的 66 个核苷 酸可形成自由能为 $-40.3 \mathrm{kcal} / \mathrm{mol}$ 的茎环结构, 该茎环结构能增加淀粉状蛋白的表达. 我们发 现，在 asyip cDNA 第 1,2 个 poly(A)信号和第 3 个 poly(A)信号之间的约 830 碱基序列中，同 样存在着复杂的二级结构, 估计这种结构也可能对 asyip 基因的蛋白表达有某种调控作用. asyip 基因 $5^{\prime}$ 端非翻译区 $\mathrm{GC}$ 含量非常丰富, 高达 $59.26 \%$ ，具有典型的持家基因启动子特征 ${ }^{[15]}$.

asyip 基因 2.7 和 $1.8 \mathrm{~kb}$ 两种转录本共享一个大的可读框, 编码一个 $26 \mathrm{ku}$ 的多肽. ASYIP 蛋白的氨基酸序列中存在两个大的强疏水区, 其 C 端含有一个内质网捕获信号(Lys-Lys-LysAla-Glu), 考虑到两个强疏水区可能是跨膜区, 推测 ASYIP 可能是一个内质网跨膜蛋白. 两个 蛋白激酶 C 磷酸化位点和两个酪蛋白激酶磷酸化位点也存在于 ASYIP 氨基酸序列中, 这 4 个 位点是否与 asyip 诱发细胞凋亡的功能有关有待进一步研究.

在细胞调亡途径中, 多个保守的蛋白功能区发挥了重要的蛋白相互作用和信号传导功能, 例如 Fas 的 DD(death domain)功能区 ${ }^{[16]}$, FADD 的 DED(death effector domain)功能区 ${ }^{[17]}$, Caspase 招募区 CARD(Caspase recruitment domain) ${ }^{[18]}$, Bcl-2 家族的 BH3(Bcl-2 homology domain 3)功 能区 ${ }^{[19]}$ 等, 通常称这些蛋白功能区为死亡相关功能区. ASYIP 和 ASY C 端的疏水性氨基酸含 量非常丰富, 在氨基酸序列上具有 56.60\%的高度同源性, 在 ASYIP 氨基酸 88 120 区和 151 187 区同源性更高, 达 88\%和 81\%, 这可能是 ASYIP 和 ASY 在酵母和哺乳动物细胞中 能相互结合的分子基础. 尽管 ASYIP 在细胞内能与 ASY 相互结合, 但 asyip 是否调控 asy 诱 发的细胞凋亡仍然不得而知, 二者在功能上的关系是需要进一步研究的内容. 对 asy 突变体的 研究发现, ASY 的 C 端对它的细胞调亡诱发功能是必须的. 将 ASYIP 和 ASY 的 C 端与目前 已知的死亡相关功能区进行比较, 并未发现任何同源性. 因此, 我们认为, ASYIP 和 ASY C 端的高度同源区不仅仅与蛋白在细胞内质网的跨膜相关, 而且与蛋白质之间的相互作用及诱 发细胞凋亡的功能相关, 可能是一个新的死亡相关功能区.

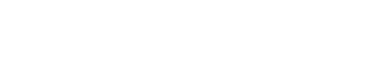

1 Tomislav D, Charles M R, Craig B T. Signal transduction pathways that regulate cell survival and cell death. Oncogene, 1998, 17: $3207 \sim 3213$

2 Shigeomi S, Masashi N, Yoshihide T, et al. Bcl-2 family proteins regulate the release of apoptogenic cytochrome c by the mitochondrial channel VDAC. Nature, 1999, 399: 483 487

3 Gabriel N, Mary A B, Hu Y M, et al. Caspases: the proteases of the apoptotic pathway. Oncogene, 1998, 17: 3 237 3 245

4 Masato E, Hideki S, Hideki Y, et al. A caspase-activated DNase that degrades DNA during apoptosis, and its inhibitor ICAD. Nature, 1998, 391: 43 50

5 Hideki S, Masato E, Shigekazu N, et al. Cleavage of CAD inhibitor in CAD activation and DNA degradation during apoptosis. 
Nature, 1998, 391: 96 99

6 Li Q, Oka K, Yoshioka N, et al. Isolation and characterization of a novel gene ASY which efficiently induces apoptosis through a leucine zipper-mediated mechanism. In: The Proceedings of the 57th Annual Meeting of the Japanese Cancer Association. Yokohama: Yokohama University Press , 1998. 148

7 Field S, Song O. A novel genetic system to detect protein-protein intractions. Nature, 1989, 340: 245 246

8 Sambrook J, Fritsch E F, Maniatis T, et al. Molecular Cloning, a Laboratory Mannual. 2nd ed. New York: Cold Spring Harbor Laboratry Press, 1989

9 Kozak M. An analysis of vertebrate mRNA sequences: intimations of translational control. J Cell Biol, 1991, 115: 887 903

10 Michael R J, Tommy N, Per A P. Retrieval of transmembrane proteins to the endoplasmic reticulum. J Cell Biol, 1993, 121: 317 333

11 Yuzuru I, Takaharu K, Akira M, et al. The CED-4-homologours protein FLASH is involved in Fas-mediated activation of caspase-8 during apoptosis. Nature, 1999, 398: 777 785

12 Hailing H, Jessie X, David V G. The TNF receptor-1-associated protein TRADD signals cell death and NF- $\kappa$ B activation. Cell, 1995, 81: 495 504

13 胡建红, 中西真人, 齐义鹏. 非编码序列对仙台病毒 $H N$ 基因表达的调控作用. 中国科学, C 辑, 1999, 29(3): 225 231

14 Sauvage F de, Kruys V, Marinx O, et al. Alternative polyadenylation of the amyloid protein precursor mRNA regulates translation. EMBO J, 1992, 11(8): 3 099 3 103

15 Macleod D, Charlton J, Mullins J, et al. Sp1 site in the mouse Aprt gene promoter are required to prevent methylation of the CpG island. Gene Dev, 1994, 8: 2 282 2 292

16 Nagata S. Apoptosis by death factor. Cell, 1997, 88: 355 365

17 Muzio M, Chinnaiyan A M, Kischkel F C, et al. FLICE, a novel FADD-homologous ICE/CED-3-like protease, is recruited to the CD95(Fas/APO-1) death-inducing signaling complex. Cell, 1996, 85: 817 827

18 Hofmann K, Bucher P, Tschopp J. The CARD domain: a new apoptotic signaling motif. Trends Biochem Sci, 1997, 22: $155 \sim 156$

19 李 燕, 齐义鹏, 肖庚富. 新基因 $b a k$ 研究进展简评. 科学通报, 1999, 44(4): 351 354 